\title{
Microconcreto de alto desempenho: propriedades e comportamento de pilar à flexão normal composta
}

\author{
Microconcrete of high performance: \\ properties and behavior under \\ combined compression and \\ one-axis bending
}

\author{
Augusto Costa Silva ${ }^{1}$, Edgar Barcarji ${ }^{2}$, Jeovan Pereira das Virgens ${ }^{2}$,
} Andressa de Andrade Tassi ${ }^{3}$

\author{
${ }^{1}$ Escola de Engenharia Civil e Ambiental, Universidade Federal de Goiás - UFG, CEP 74605-220, Goiânia, GO, Brasil. \\ e-mail: augustocscivil@gmail.com.br \\ ${ }^{2}$ Escola de Engenharia Civil e Ambiental, Universidade Federal de Goiás - UFG, CEP 74605-220, Goiânia, GO, Brasil. \\ e-mail: edgarbacarji@hotmail.com; jeovan2001@yahoo.com.br \\ ${ }^{3}$ Stc soluções para construção civil ltda, CEP 74563-430, Goiânia, GO. \\ e-mail: andressatassi@hotmail.com
}

\section{RESUMO}

O microconcreto de alto desempenho é um material que possui dimensão máxima de agregado reduzida e baixa relação água/aglomerante (a/aglm). Com o objetivo de verificar a aplicabilidade em elementos estruturais, estudou-se o comportamento do material e de um pilar executado com microconcreto de alto desempenho. Como referência, estudou-se outro concreto com mesmas proporções de materiais, apenas variando a dimensão máxima do agregado. Avaliaram-se as propriedades no estado fresco, mecânicas e de durabilidade dos materiais. Quanto aos pilares, os mesmos foram submetidos a uma situação de projeto de flexão normal composta. Estes pilares foram instrumentados por meio de extensômetros elétricos de resistência, relógios comparadores e Linear Variable Differential Transformer (LVDT), de forma a obter os deslocamentos horizontais, deformações na face comprimida do concreto e as deformações nas armaduras de aço; também foram acompanhadas as aberturas de fissuras por meio de marcação com pincel atômico. Quanto às propriedades no estado fresco, mecânicas e de durabilidade, verificou-se que o microconcreto teve melhor desempenho em relação ao concreto de referência, desempenho este atribuído às melhores propriedades da zona de interface pasta-agregado. O pilar de microconcreto rompeu por esmagamento do concreto da face comprimida, na região intermediária do pilar. Já o pilar de concreto convencional rompeu numa seção acima da intermediária, e a ruptura ficou caracterizada pelo desplacamento do concreto em duas faces adjacentes, em forma de quina, sugerindo uma ruptura por flexão composta oblíqua. Fez-se a comparação dos resultados experimentais obtidos com os resultados obtidos pelo software Esbelt 2.1, que considera os módulos de elasticidade segundo a NBR 6118 (ABNT, 2003). Ao final, pode-se constatar o melhor comportamento do pilar de microconcreto em relação ao pilar de concreto convencional, em termos de maior rigidez, sugerindo vantagens na aplicação do material em elementos estruturais destinados à pré-fabricação de elementos com seções transversais delgadas.

Palavras-chave: Microconcreto, propriedades mecânicas, permeabilidade, pilares.

\section{ABSTRACT}

The high-performance microconcrete is a material that has reduced maximum characteristic dimension aggregate and low water / cement ratio (w/c). In order to verify the applicability in structural elements, the behavior of the material and of a pillar executed with high performance microconcrete were studied. As reference, another concrete with the same proportions of materials was studied, only varying the maximum size of the aggregate. The fresh state, mechanical and durability properties of the materials were evaluated. As for the pillars, they were subjected to a project situation of combined compression and one-axis bending. Considering the columns, in order to obtain the horizontal displacements, deformations in the compressed face of 
the concrete and the strain in the bars, these columns were instrumented by means of strain gages, comparator watches and Linear Variable Differential Transformer (LVDT); The apertures of cracks were also monitored by means of atomic brush marking. As for the properties in the fresh state, mechanical and durability, it was verified that the microconcrete had better performance in relation to the reference concrete that was attributed to the best properties of the interfacial transition zone. The microconcrete column ruptured by crushing concrete from the compressed face in the intermediate region of the column. The conventional concrete column broke in a section above the intermediate one, and the rupture was characterized by the displacement of the concrete in two adjacent shaped faces, suggesting a rupture by oblique combined bending. The results obtained were compared with the results obtained by the software Esbelt 2.1, which considers the modulus of elasticity according to the standard NBR 6118 (ABNT, 2003). At the end, it was possible to observe the best behavior of the microconcrete column in relation to the conventional concrete column, in terms of greater rigidity, suggesting applicability of the microconcrete in structural elements like precasting elements with thin cross-sections.

Keywords: Microconcrete, Mechanical properties, permeability, column.

\section{INTRODUÇÃO}

Segundo CAMPOS [13], a industrialização da construção constitui-se no desenvolvimento dos produtos e processos tecnológicos. Em termos de produtos, com o avanço da tecnologia do concreto foi possível obter concretos mais resistentes e duráveis, sendo que, usados em elementos estruturais possibilitam a redução das seções transversais, gerando economia de material e redução de custos. Os produtos, quando utilizados em elementos pré-fabricados, devem facilitar os processos de produção, tais como a montagem e o transporte. Devem também adequar-se aos meios de produção disponíveis nos centros urbanos e as características regionais. A expressão alto desempenho refere-se à qualidade da matriz, que proporciona elevada resistência mecânica e baixa porosidade ao concreto.

O microconcreto pode ser definido como um concreto de alto desempenho proporcionado com microagregados cujo tamanho das partículas varia cerca de $0,5 \mathrm{~mm}$ a menos de $1 \mu \mathrm{m}$. A incorporação destes microagregados melhora a densidade de empacotamento de partículas do sistema cimentício, aprimorando, assim, as propriedades reológicas e mecânicas do material [15]. De acordo com FELEKOĞLU [14], se a viabilidade e a adequação técnica forem verificadas, a tendência é de se aumentar a quantidade de micro-agregados em um sistema de concreto podendo eliminar o uso de agregado graúdo. A principal característica que diferencia o microconcreto do concreto convencional é a dimensão máxima do agregado graúdo. Para o microconcreto, este parâmetro possui valores menores que os utilizados no concreto convencional ou não possui agregado graúdo. Assim, a curva tensão deformação pode apresentar formato distinto para os dois materiais para um mesmo nível de resistência à compressão. Dessa forma, esta variável pode influenciar significantemente o comportamento de pilares, que são os elementos que absorvem as principais cargas atuantes na estrutura. Além da resistência à compressão, o módulo de elasticidade, que denota a rigidez do material, é considerado como uma das propriedades mais importantes do concreto. Como componente principal com alta relação de volume, o agregado obviamente tem grande influência no valor do módulo. Em outro aspecto, a zona de transição também tem um impacto notável no módulo de elasticidade [14].

SCRIVENER et al. [20] propuseram que a discrepância de tamanho entre grãos de cimento e agregados provavelmente induz a uma zona de transição interfacial com a região da pasta de cimento em torno das partículas dos agregados, uma área com um excesso de porosidade. Outra origem do aumento da porosidade pode se dar também pelo acumulo de água livre ao redor da superfície do agregado, especialmente sobre agregados graúdos [16]. De acordo com seu mecanismo de formação, a zona de transição é considerada afetada por fatores como relação água / cimento, textura do agregado e distribuição do seu tamanho. A maioria das pesquisas existentes indica que o tamanho do agregado pode afetar a propriedade da zona de transição, sendo que um agregado maior geralmente resulta em uma zona de transição mais porosa e com menor força de ligação [21]. De acordo com SCRIVENER et al. [20], métodos de modificar a zona de transição são fundamentais no desenvolvimento de concreto de alto desempenho. A forma mais eficaz de modificar a zona transição é adicionar uma proporção de partículas finas, como a sílica ativa, que pode empacotar mais a superfície do agregado. O seu impacto na zona de transição é de significância considerável, levando ao aumento da ductilidade entre outros efeitos.

Segundo SAHMARAN et al. [19], as misturas de concreto de alto desempenho com tamanhos dos agregados máximos menores ganharam popularidade e geralmente são escolhidas pela facilidade de preencher os elementos estruturais. Portanto, entender o comportamento destes elementos confeccionados com o microconcreto de alto desempenho, torna-se fator importante na análise estrutural. 
Como esclarece BORGES [12], em se tratando de concretos de alto desempenho as dimensões de pilares podem ser reduzidas. Converge-se, então, para o caso de pilares mais esbeltos, onde se torna necessário o estudo dos efeitos de segunda ordem. Devido ao avanço tecnológico na área computacional, os projetistas dispõem de softwares capazes de calcular os pilares por diversos métodos, inclusive o obrigatório para estruturas esbeltas, o Método Geral. Dessa forma, pode-se optar por sua utilização em substituição aos métodos simplificados, mesmo em pilares de mediana esbeltez.

O objetivo geral deste trabalho foi contribuir no estudo do microconcreto de alto desempenho, considerando suas propriedades no estado fresco e endurecido, bem como considerando o comportamento de um pilar executado com este material. Como objetivos específicos podem-se destacar:

- Avaliar as propriedades de trabalhabilidade por meio do ensaio do abatimento do tronco de cone;

- Avaliar as propriedades mecânicas como resistência à compressão, resistência à tração e módulo de elasticidade;

- Qualificar o concreto quanto à durabilidade, por meio dos ensaios de absorção total de água e índice de vazios;

- Estudar o comportamento, em termos de diagramas carga deslocamento, carga deformação no concreto e nas barras de armaduras, de um pilar de microconcreto de alto desempenho submetido à flexão normal composta, em função da redução da granulometria dos agregados.

\section{MATERIAIS E MÉTODOS}

Neste tópico são apresentados os dados relativos ao microconcreto e ao concreto de referência utilizados na confecção dos pilares. São definidas as características geométricas, descritos os procedimentos preparatórios dos pilares e o procedimento de execução do ensaio dos pilares.

\subsection{Concretos}

Para a produção dos concretos utilizaram-se como aglomerante o cimento portland branco estrutural CPB-40 e a sílica ativa. Quanto aos agregados, utilizaram-se areia de rio como agregado miúdo e brita de origem granítica como agregado graúdo, com diferente granulometria para o microconcreto e o concreto de referência. Além disso, utilizaram-se água potável e aditivo superplastificante à base de policarboxilatos.

$\mathrm{O}$ valor da relação a/aglom obtido pelo ajuste de dosagem foi igual a 0,4 com um teor de superplastificante igual a $0,35 \%$ em relação à massa do aglomerante. As proporções aglomerante: areia: brita, em massa, foi de 1,0:1,5:1,5. Na Tabela 1 são apresentados os consumos de materiais por $\mathrm{m}^{3}$ de concreto, observando-se uma estrutura granular muito semelhante, variando-se apenas a dimensão máxima do agregado graúdo dos concretos.

Tabela 1: Consumo de materiais $\left(\mathrm{kg} / \mathrm{m}^{3}\right)$.

\begin{tabular}{l|l|l}
\hline MATERIAL & MICROCONCRETO & REFERÊNCIA \\
\hline Cimento & 478,40 & 481,19 \\
Sílica Ativa & 47,84 & 48,12 \\
\hline Areia & 789,35 & 793,96 \\
\hline Brita $9,5 \mathrm{~mm}$ & 789,35 & - \\
Brita $12,5 \mathrm{~mm}$ & - & 396,98 \\
Brita $19,0 \mathrm{~mm}$ & - & 396,98 \\
\hline Água & 210,49 & 211,72 \\
\hline Superplastificante & 1,84 & 1,85 \\
\hline
\end{tabular}

Foram avaliadas as propriedades no ensaio fresco e endurecido, como detalhadas na Tabela 2.

Todos estes ensaios, realizados na idade de 28 dias. Utilizaram-se 3 corpos de prova cilíndricos para cada ensaio, moldados nas dimensões $10 \mathrm{~cm}$ x $20 \mathrm{~cm}$. Os corpos de prova foram submetidos à cura em câmara úmida, onde permaneceram até um dia antes da realização dos ensaios. 
Tabela 2: Ensaios realizado no estado fresco e endurecido, seguindo-se as prescrições em normas.

\begin{tabular}{l|l}
\hline ENSAIOS & NORMAS \\
\hline Densidade e o teor de ar incorporado & NBR 9833 [8] \\
\hline Abatimento do tronco de cone & NBR NM 67 [9] \\
\hline Resistência à compressão & NBR 5739 [1] \\
\hline Módulo de elasticidade & NBR 8522 [6] \\
\hline Tração por compressão diametral & NBR 7222 [5] \\
\hline $\begin{array}{l}\text { Absorção total de água e índice de } \\
\text { vazios }\end{array}$ & NBR 9778 [7] \\
\hline
\end{tabular}

\subsection{Aço para armadura}

Para as armaduras longitudinais foram utilizadas barras de aço do tipo CA-50, com diâmetro nominal de 10 $\mathrm{mm}$. Quanto às armaduras transversais, foram utilizadas barras do tipo CA-60, com diâmetro nominal de 5 $\mathrm{mm}$. Para estas barras, realizaram-se os ensaios de caracterização para determinação das tensões de escoamento $\left(f_{y}\right)$ e última $\left(f_{u}\right)$, módulo de elasticidade $\left(\mathrm{E}_{\mathrm{s}}\right)$ e deformação de escoamento $\left(\varepsilon_{\mathrm{u}}\right)$, seguindo as prescrições da NBR ISO 6892-1 [4]. Os resultados da caracterização das armaduras são apresentados na Tabela 3.

Tabela 3: Caracterização das armaduras.

\begin{tabular}{l|l|l|l|l}
\hline \multicolumn{6}{l}{ CARACTERIZAÇÃO DO AÇO PARA ARMADURAS } \\
\hline \multicolumn{1}{c|}{ DIÂMETRO (MM) } & FY (MPA) & FU (MPA) & EU (MM/M) & ES (GPA) \\
\hline 5 & 720,88 & 754,83 & 3,55 & 202,93 \\
\hline 10 & 596,53 & 717,42 & 2,86 & 208,21 \\
\hline
\end{tabular}

\subsection{Características gerais dos pilares}

Os modelos de pilares ensaiados foram identificados como P-REF e P-MIC, para o pilar confeccionado com o concreto de referência e para o pilar confeccionado com o microconcreto, respectivamente.

O modelo dos pilares que foram ensaiados apresenta dois consolos, um na base e outro no topo. Isto para permitir a aplicação de força excêntrica ao pilar, provocando momento fletor na região central. Adotouse a seção transversal de $120 \mathrm{~mm}$ x $125 \mathrm{~mm}$ e altura do pilar igual a $2000 \mathrm{~mm}$, para ambos os modelos, sendo submetidos à excentricidade inicial de carregamento de $40 \mathrm{~mm}$. O detalhamento da armadura de ambos os pilares ensaiados é apresentado na Figura 1.
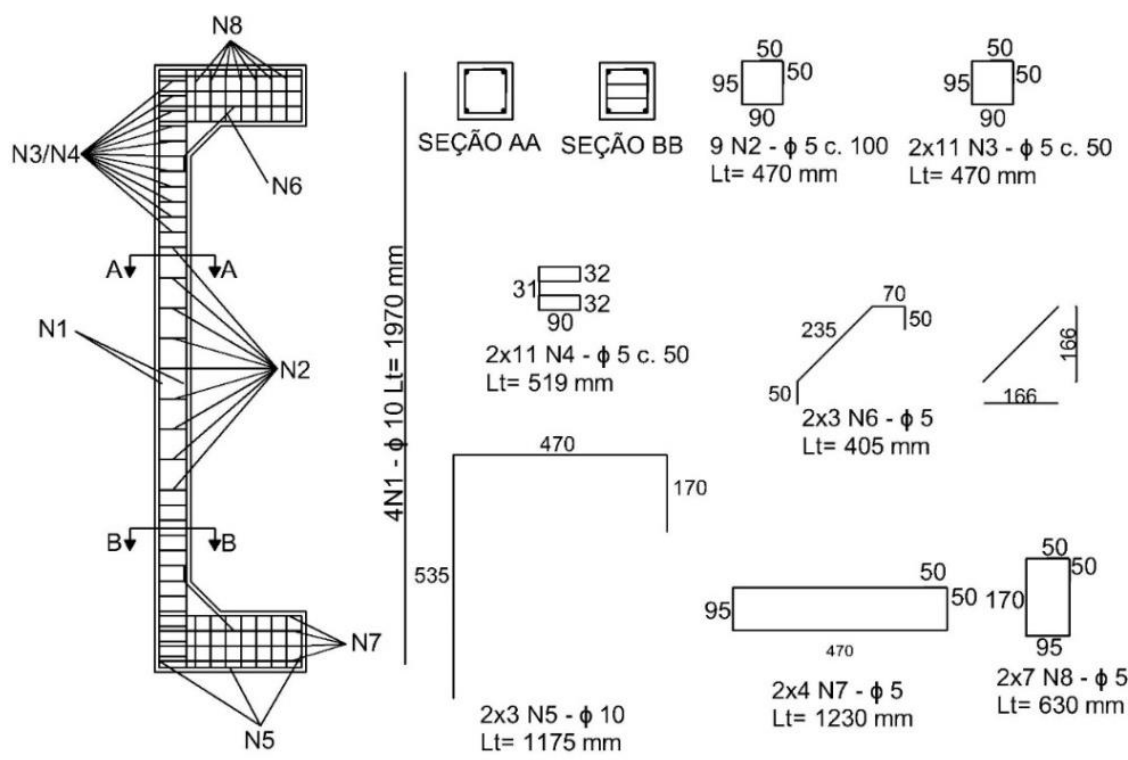

Figura 1: Detalhamento da armadura dos modelos P-MIC e P-REF. (mm) 


\subsection{Instrumentação}

Realizou-se a instrumentação das quatro barras longitudinais, por meio de extensômetros elétricos de resistência (EER). Em cada barra foram colocados EER do lado externo das mesmas. Por meio desses extensômetros foram medidas as deformações sofridas pelas barras. Os extensômetros elétricos de resistência utilizados foram da marca Excel sensores. Estes são do tipo PA-06-250BA-120L. Em relação às barras transversais, por não haver carregamento horizontal, elas não foram monitoradas. A Figura 2 apresenta o detalhe da instrumentação das barras longitudinais dos modelos.

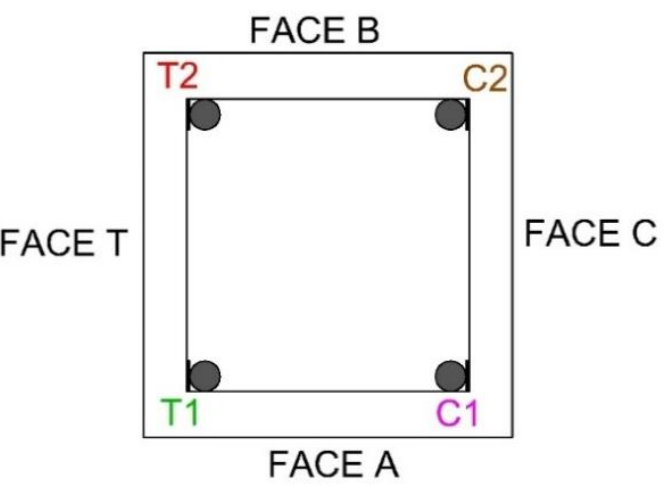

a) Detalhe de instrumentação da armadura.

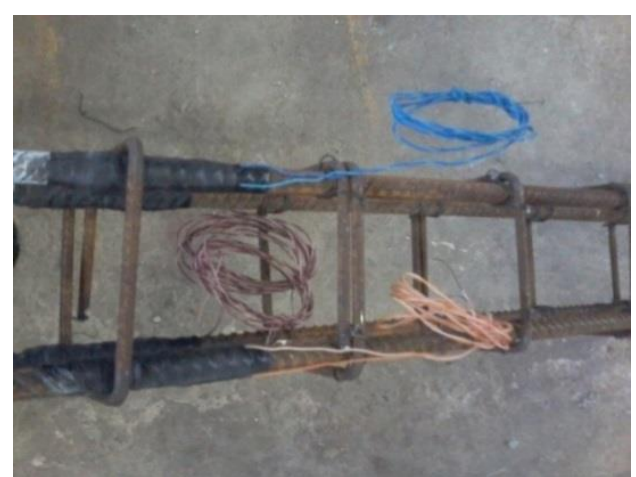

b) Armaduras com instrumentação finalizada.

Figura 2: Instrumentação nas barras das armaduras longitudinais.

$\mathrm{Na}$ instrumentação do concreto, para a determinação das deformações de compressão, foram utilizados três extensômetros elétricos de resistência, do tipo PA-06-201BA-120-L, na região central dos pilares, postos na face comprimida (Face C). O posicionamento dos EER na Face $\mathrm{C}$ foi realizado conforme a Figura $3 \mathrm{a}$.

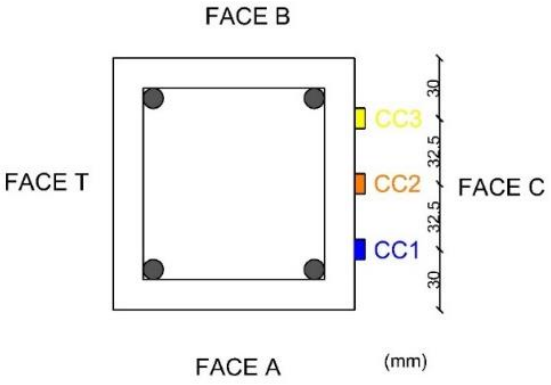

a) Posição dos EER na face comprimida do concreto.

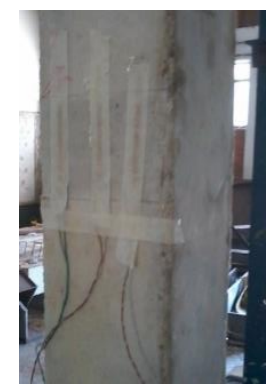

b) Instrumentação da face $\mathrm{C}$ do concreto.

Figura 3: Instrumentação das deformações no concreto, na região central da face $\mathrm{C}$ do pilar

Para medir os deslocamentos horizontais, utilizaram-se cinco relógios comparadores digitais, com precisão de 0,01 mm, acoplados em bases magnéticas e um Linear Variable Differential Transformer (LVDT) na região central dos pilares, conforme pode ser observado na Figura 4. Para a fixação dos relógios comparadores foi utilizado um suporte metálico, colocado atrás do pórtico de reação. 


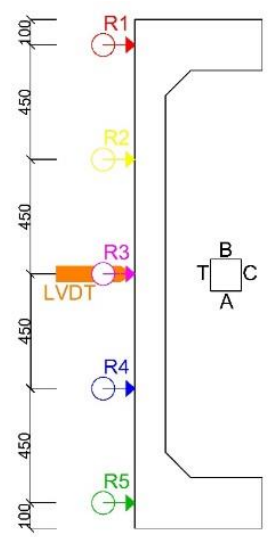

Figura 4: Instrumentação com relógios comparadores e LVDT.

O desenvolvimento das fissuras na face tracionada dos modelos (Face $\mathrm{T}$ ) foi registrado por meio de marcações com pincel atômico, a cada incremento de carga.

\subsection{Ensaio dos pilares}

Para realização dos ensaios, os pilares foram posicionados na posição vertical com auxílio da ponte rolante e talha mecânica; após posicionado o pilar, foi posicionado o atuador hidráulico no consolo inferior, sendo a célula de carga posicionada no consolo superior.

\section{RESULTADOS E DISCUSSÕES}

São discutidos neste item os resultados das propriedades no estado fresco, propriedades mecânicas e de durabilidade dos concretos e os resultados obtidos nos ensaios dos pilares, tais como: diagramas carga $\mathrm{x}$ deformação nas armaduras e na face comprimida dos concretos, curva carga x deslocamentos para cada modelo e aberturas das fissuras.

\subsection{Propriedades no estado fresco, mecânicas e de durabilidade dos concretos}

Quanto ao concreto de referência, obtiveram-se os seguintes valores para o abatimento, teor de ar incorporado e densidade, respectivamente: $165 \mathrm{~mm}, 2,1 \%$ e $2.329 \mathrm{~kg} / \mathrm{m}^{3}$. No que se refere ao microconcreto, foram obtidos os valores: $160 \mathrm{~mm}, 2,4 \%$ e $2.315 \mathrm{~kg} / \mathrm{m}^{3}$. Assim, observam-se características muito próximas quanto à trabalhabilidade, medida pelo Slump test entre os dois concretos. Quanto ao teor de ar incorporado, o microconcreto apresentou valor ligeiramente superior (14\%) e densidade ligeiramente inferior $(0,6 \%)$ em relação ao concreto de referência.

Na Tabela 4 são apresentados os valores médios obtidos para a resistência à compressão $\left(\mathrm{f}_{\mathrm{c}}\right)$, módulo de elasticidade $\left(\mathrm{E}_{\mathrm{cs}}\right)$, resistência à tração por compressão diametral $\left(\mathrm{f}_{\mathrm{tcd}}\right)$, absorção total de água (Abs) e índice de vazios $\left(\mathrm{I}_{\mathrm{v}}\right)$ para os dois concretos estudados. Os valores entre parêntesis referem-se aos desvios padrões.

Tabela 4: Propriedades mecânicas e de durabilidade dos concretos.

\begin{tabular}{l|l|l|l|l|l}
\hline CONCRETO & F $_{\mathrm{C}}$ (MPA) & $\mathrm{E}_{\mathrm{CS}}(\mathrm{GPA})$ & $\mathrm{F}_{\mathrm{TCD}}$ (MPA) & ABS (\%) & $\mathbf{I}_{\mathbf{V}}(\%)$ \\
\hline REF & $52,7(3,5)$ & $32,1(1,2)$ & $4,73(0,63)$ & $5,97(0,33)$ & $12,67(0,63)$ \\
\hline MIC & $56,4(5,7)$ & $34,8(1,5)$ & $5,81(0,52)$ & $5,61(0,12)$ & $11,76(0,22)$ \\
\hline
\end{tabular}

Observa-se que o valor da resistência média à compressão obtida para o microconcreto foi cerca de $7 \%$ superior à do concreto de referência. Todavia, pela análise do desvio padrão, verifica-se uma faixa de resistências comuns aos dois concretos, o que não permite assegurar que estes dois concretos apresentam resistências estatisticamente diferentes.

O valor médio do módulo de elasticidade obtido para o microconcreto foi cerca de $8 \%$ superior ao do concreto de referência. Pela análise do desvio padrão, verifica-se não existir uma faixa de valores de módulo de elasticidade que compreende os dois materiais, sugerindo que o microconcreto possui módulo de elastici- 
dade estatisticamente maior que o concreto de referência.

O valor do módulo de elasticidade secante foi calculado conforme a NBR 6118 [3], para comparação com os resultados experimentais. A razão entre o módulo de elasticidade teórico, obtido pela norma, e o experimental foi igual a 1,05 para o microconcreto. Esta mesma razão, em se tratando do concreto de referência, foi igual a 1,08, conforme é apresentado na Tabela 5. Assim, observa-se que a equação sugerida pela referida norma forneceu valores do módulo de elasticidade ligeiramente superiores em relação aos os concretos estudados. Apresentam-se ainda na Tabela 5 os valores estimados do módulo de elasticidade segundo a versão de 2003 da NBR 6118 que, neste caso, melhor se aproximou dos resultados experimentais. Esta informação é importante pois nela se baseia o software Esbelt 2.1 utilizado na análise dos pilares.

Tabela 5: Comparação dos módulos de elasticidade teóricos e experimental.

\begin{tabular}{l|l|l|l|l|l|l}
\hline CONCRETO & ROCHA & $\boldsymbol{\alpha}_{\mathrm{E}}$ & $\begin{array}{l}\mathrm{E}_{\text {CS NBR 6118- }} \\
\mathbf{2 0 1 4} \text { (GPA) }\end{array}$ & $\begin{array}{l}\mathrm{E}_{\text {CS NBR 6118- }} \\
\mathbf{2 0 0 3} \text { (GPA) }\end{array}$ & $\begin{array}{l}\mathrm{E}_{\text {CS }} \text { 6118- } \\
\text { 2014/EEXP }\end{array}$ & $\begin{array}{l}\mathrm{E}_{\text {CS }} \text { 6118- } \\
\text { 2003/EEXP }\end{array}$ \\
\hline Microconcreto & Granito & 1,0 & 36,55 & 33,60 & 1,05 & 0,97 \\
\hline Referência & Granito & 1,0 & 34,81 & 32,33 & 1,08 & 1,01 \\
\hline
\end{tabular}

Quanto a resistência à tração por compressão diametral, também se obteve um valor médio para o microconcreto superior $(22,8 \%)$ ao do concreto de referência, embora, pelo desvio padrão obtido, não se possa afirmar que houve uma diferença significativa.

Nas demais propriedades analisadas, relacionadas à durabilidade dos concretos, também se observa o melhor desempenho do microconcreto em relação ao concreto de referência: absorção total de água e índice de vazios $6 \%$ e 7,2\% inferiores, respectivamente. Observa-se que, para esta última propriedade, não se observa uma faixa comum de valores quando se considera o desvio padrão.

Tais resultados são corroborados pelas pesquisas de SCRIVENER et al. [20], ROHDEN [18], NETO et al. [17] e LAGERBLAD e KJELLSEN [16], os quais apontaram para o melhor desempenho do concreto com agregados graúdos de menor diâmetro, explicado por melhores propriedades da zona de transição pastaagregado, por menores microfissuras existentes e por redução da água livre, quando comparadas ao mesmo concreto com agregados de maiores dimensões. Como consequência, houve uma redução da interconectividade dos poros, reduzindo a absorção de água e o índice de vazios. Assim, as dimensões menores dos agregados no microconcreto, promoveram ganhos, tanto nas propriedades mecânicas, quanto na redução da permeabilidade do concreto.

\subsection{Ensaio de pilares}

Realizaram-se os ensaios dos dois pilares submetidos a flexão normal composta nos quais foram obtidos os deslocamentos, deformações e carga última. Para se ter uma noção da ordem de grandeza da carga de ruptura e deslocamento máximo, foi feita uma análise por meio do software comercial Esbelt 2.1 onde se adotou uma resistência característica de $50 \mathrm{MPa}$, valor este muito próximo das resistências mínimas dos dois pilares analisados (ver Tabela 4). No software, os módulos de elasticidade dos materiais são estimados segundo a NBR 6118 [2], ou seja, na versão de 2003. Os coeficientes de minoração das resistências foram adotados iguais a 1,0 para o concreto e o aço e o método utilizado para a verificação da estabilidade foi o Método Geral. Ainda pelo software, obtiveram-se os seguintes resultados para uma excentricidade inicial de 40mm: Carga de ruptura de $270 \mathrm{kN}$ e deslocamento máximo de $12 \mathrm{~mm}$.

Quanto aos ensaios, a ruptura no P-MIC se deu na carga de $305 \mathrm{kN}$, sendo a ruptura localizada na seção intermediária do pilar. A ruptura do pilar P-REF se deu na carga de $192,5 \mathrm{kN}$, fora da seção transversal intermediária, na região a qual estava posicionado o relógio comparador R2 (cerca de $45 \mathrm{~cm}$ acima da região central do pilar). Observou-se nesta região o desplacamento parcial do concreto nas faces C e A.

As relações entre as cargas de ruptura observadas experimentalmente e a obtida no software Esbelt 2.1 foram 1,13 e 0,70 para o P-MIC e P-REF, respectivamente. A relação maior que 1,0 para o P-MIC é razoável (13\% superior ao valor teórico), uma vez que, via de regra, os métodos teóricos são mais conservadores em relação aos resultados experimentais. Já para o P-REF, obteve-se uma relação inferior a 1,0 (30\% inferior ao valor teórico), o que sugere uma ruptura precoce para este pilar. Uma possibilidade para esta ruptura precoce pode ter sido o surgimento de excentricidade acidental na direção paralela ao plano de aplicação de carga. Esta excentricidade acidental pode ter surgido pelo desalinhamento das placas de apoio inferior e/ou superior do pilar. 


\subsection{Deslocamentos horizontais}

As medições dos deslocamentos com os relógios comparadores foram feitas até as cargas de $200 \mathrm{kN}$ e 180 $\mathrm{kN}$ para o P-MIC e para o P-REF, respectivamente. A medição dos deslocamentos horizontais por meio do LVDT se deu até a carga de ruptura dos modelos. A fim de se comparar o desenvolvimento dos deslocamentos horizontais dos pilares fizeram-se as relações entre deslocamento horizontal em R1 e R5 (R1/R5) e entre R2 e R4 (R2/R4) (ver Figura 4) para ambos os pilares. Estes valores são dados na Tabela 6.

Tabela 6: Razão entre os deslocamentos em posições simétricas, para P-MIC e P-REF.

\begin{tabular}{l|l|l|l|l}
\hline & \multicolumn{3}{l}{ P-REF } & \multicolumn{2}{l}{ P-MIC } \\
\hline CARGA (KN) & R1/R5 & R2/R4 & R1/R5 & R2/R4 \\
\hline 10,0 & 0,80 & 0,86 & - & - \\
\hline 20,0 & 0.80 & 1,95 & - & 2,00 \\
\hline 40,0 & 0,79 & 1,36 & 1,57 & 1,22 \\
\hline 60,0 & 0,81 & 1,59 & 1,25 & 1,14 \\
\hline 80,0 & 0,62 & 0,98 & 1,32 & 1,10 \\
\hline 100,0 & 0,63 & 0,75 & 1,24 & 1,08 \\
\hline 120,0 & 1,25 & 0,77 & 1,31 & 1,05 \\
\hline 140,0 & 0,59 & 0,67 & 1,33 & 1,08 \\
\hline 160,0 & 0,61 & 0,80 & 1,33 & 1,10 \\
\hline 180,0 & 0,69 & 0,72 & 1,44 & 1,13 \\
\hline 200,0 & - & - & 1,47 & 1,12 \\
\hline
\end{tabular}

A grande maioria destes resultados demonstra que, para o pilar P-REF, os deslocamentos da parte superior (nas regiões de R1 e R2) do pilar foram menores que os deslocamentos da parte inferior (nas regiões de R5 e R4). Por exemplo, para este pilar a maioria das relações R1/R5 e R2/R4 é menor do que 1,0, especialmente para os carregamentos acima de 60 kN. Já para o pilar P-MIC ocorreu o inverso, ou seja, os deslocamentos da parte superior do pilar foram maiores que os deslocamentos da parte inferior, ou seja, relações R1/R5 e R2/R4 maiores do que 1,0. Uma possível causa destes comportamentos distintos pode estar numa eventual diferença de prumo dos pilares no momento da realização dos ensaios. Estes comportamentos distintos podem ser observados também nas Figura 5 e Figura 6, respectivamente, para o P-MIC e o P-REF, onde se mostram os deslocamentos horizontais dos pilares ao longo da altura.

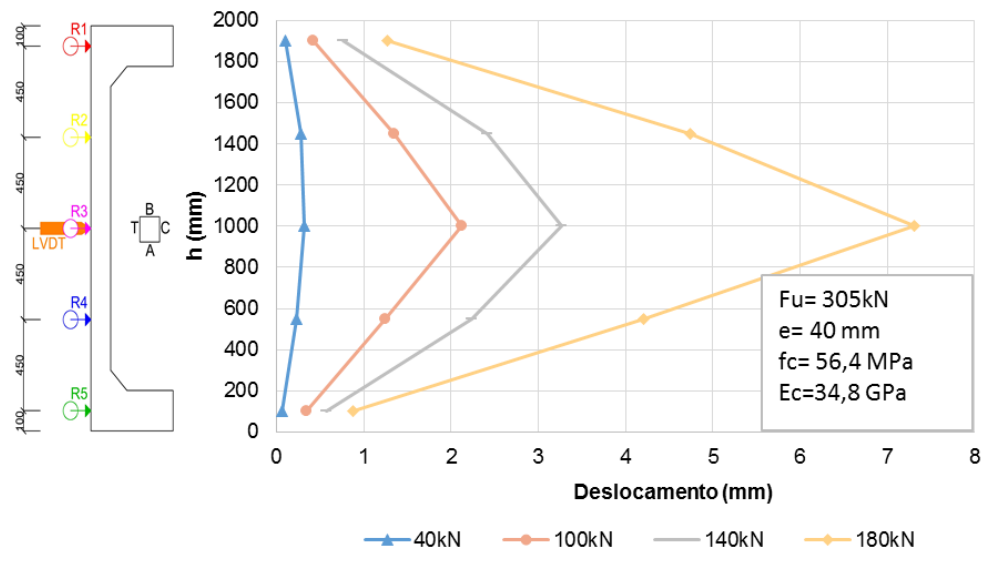

Figura 5: Deslocamentos horizontais ao longo da altura do modelo P-MIC. 


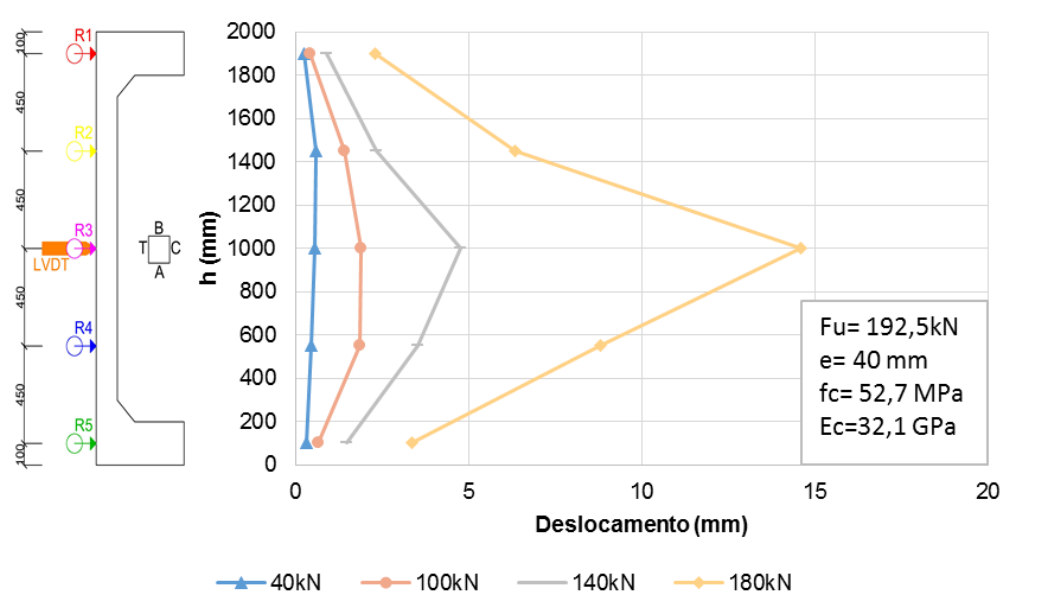

Figura 6: Deslocamentos horizontais ao longo da altura do modelo P-REF.

A Figura 7 apresenta os diagramas de carga e deslocamento na seção intermediária para os pilares PMIC e P-REF, obtidos pelo LVDT.

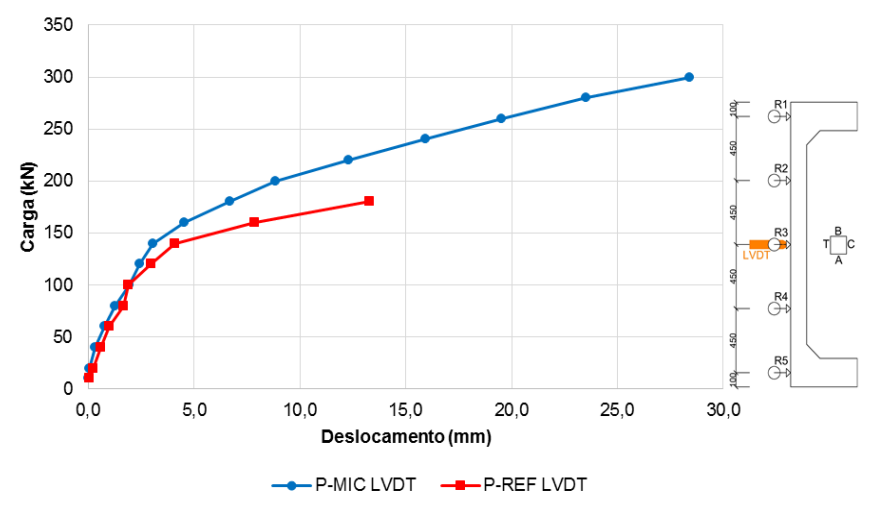

Figura 7: Diagrama Carga x Deslocamento para os modelos P-MIC e P-REF, na posição do LVDT.

Observando-se o gráfico da Figura 7 verifica-se que, para os níveis de cargas comuns aos dois pilares, à exceção da carga de $100 \mathrm{kN}$, o P-REF apresentou maiores deslocamentos. Para as cargas de $220 \mathrm{kN}$ e $180 \mathrm{kN}$, os deslocamentos correspondentes foram de 12,29 mm e 13,28 mm, para o P-MIC e P-REF, respectivamente; valores estes já maiores que o deslocamento último obtido pelo Esbelt 2.1, qual seja, de $12 \mathrm{~mm}$. Esta diferença entre o comportamento simulado pela formulação computacional e o comportamento experimental provavelmente ocorreu em função das hipóteses de cálculo implícitas no primeiro caso, geralmente mais conservadoras, como foi mostrado nos estudos de BACARJI [11].

\subsection{Deformações no concreto}

As deformações na face do concreto foram medidas por meio de extensômetros elétricos de resistência nas posições CC1, CC2 e CC3, sendo comparadas para os pilares P-MIC e P-REF por meio do diagrama carga deformação em cada posição.

A Figura 8, Figura 9 e Figura 10 apresentam os diagramas carga deformação no concreto de forma a comparar o comportamento das curvas medidas por meio de EER, nas posições CC1, CC2 e CC3, respectivamente. 


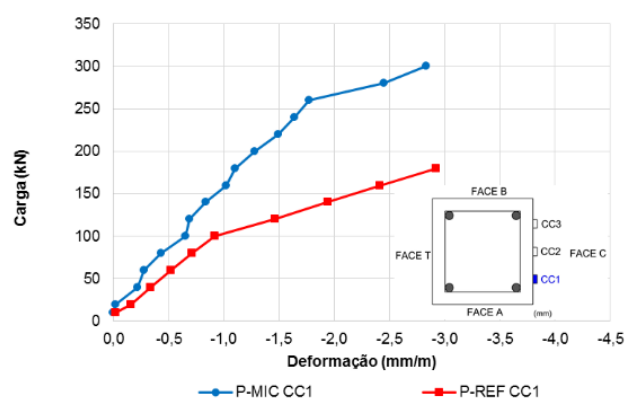

Figura 8: Comparação do comportamento Carga x Deformação no concreto do P-MIC e P-REF, na posição do EER CC1.

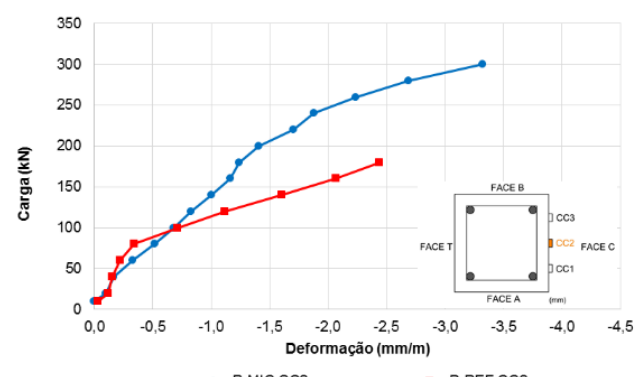

Figura 9: Comparação do comportamento Carga x Deformação no concreto do P-MIC e P-REF, na posição do EER $\mathrm{CC} 2$.

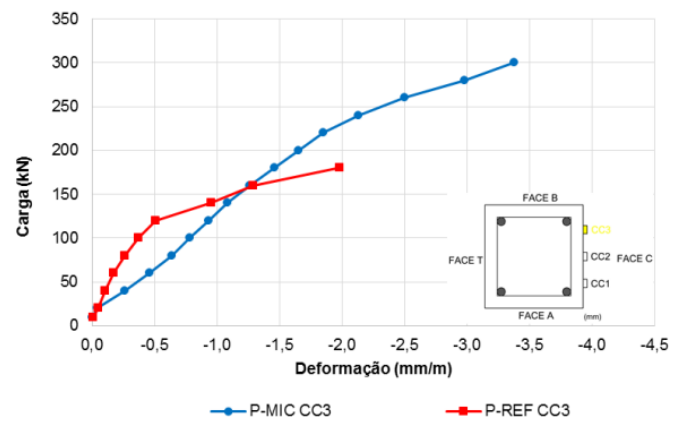

Figura 10: Comparação do comportamento Carga x Deformação no concreto do P-MIC e P-REF, na posição do EER CC3.

Na posição do extensômetro CC1, observa-se que em todos os incrementos de carga, as deformações no pilar P-REF foram superiores às deformações do pilar P-MIC; Na posição do extensômetro CC2, observase um intervalo de carga, entre 40 e $100 \mathrm{kN}$, no qual as deformações do P-REF são inferiores em relação ao PMIC; já na posição do extensômetro CC3 as deformações no P-REF são inferiores até a carga abaixo de $160 \mathrm{kN}$, o que sugere um maior nível de tensões na região do extensômetro CC1.

Ademais, para a carga de $180 \mathrm{kN}$, as deformações nos extensômetros CC1, CC2 e CC3 para o pilar PREF foram de $-2,92 \mathrm{~mm} / \mathrm{m},-2,44 \mathrm{~mm} / \mathrm{m}$ e $-1,98 \mathrm{~mm} / \mathrm{m}$. A relação entre o maior valor e o menor valor foi de 1,47. Estas deformações no pilar P-MIC foram de $-1,10 \mathrm{~mm} / \mathrm{m},-1,24 \mathrm{~mm} / \mathrm{m}$ e $-1,46 \mathrm{~mm} / \mathrm{m}$ com a relação entre o maior valor e o menor valor de 1,33. Tais relações indicam que o P-REF se afastou mais da flexão normal composta do que o P-MIC.

As deformações últimas para o concreto, segundo a NBR 6118 [3] e ACI318 [10] são, respectivamente, $-3,5 \mathrm{~mm} / \mathrm{m}$ e $-3 \mathrm{~mm} / \mathrm{m}$. Quanto ao pilar P-REF, nenhum dos extensômetros alcançou estes limites, uma vez que a ruptura não se deu nesta seção. A deformação mais próxima destes limites foi verificada no extensômetro CC1. Para o pilar P-MIC, as deformações últimas nos extensômetros CC2 e CC3 ficaram entre -3 
$\mathrm{mm} / \mathrm{m}$ e $-3,5 \mathrm{~mm} / \mathrm{m}$ e a deformação no extensômetro $\mathrm{CC} 1$ ficou um pouco abaixo, em $-2,82 \mathrm{~mm} / \mathrm{m}$.

\subsection{Deformação na armadura}

Observando-se os gráficos das Figura 11 e Figura 12 verificam-se que as deformações últimas de compressão para o P-MIC tiveram mesma ordem de grandeza, o mesmo ocorrendo nas armaduras de tração. No pilar PREF, tal semelhança não ocorre, o que também mostra o maior afastamento da flexão normal composta para este pilar.

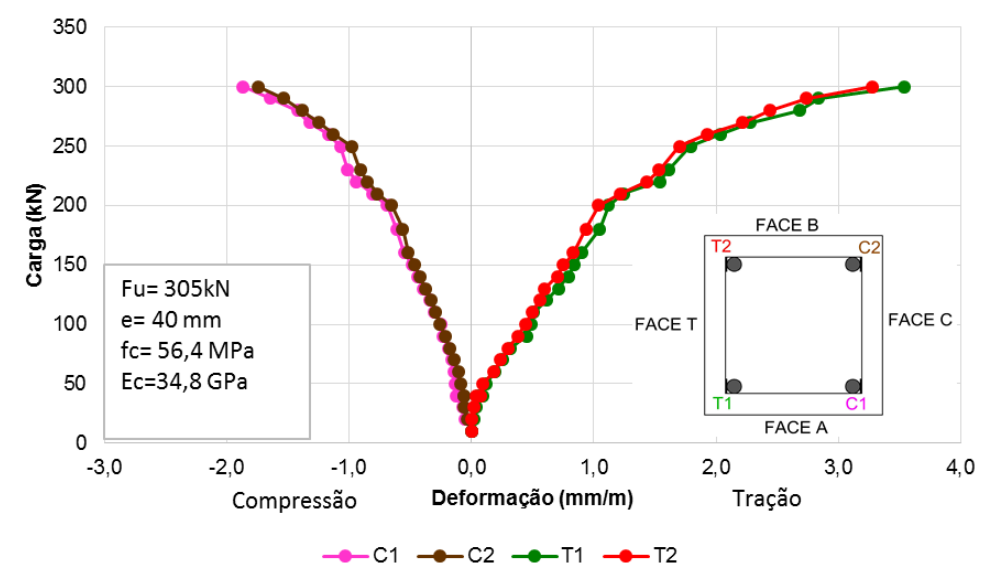

Figura 11: Diagrama Carga x Deformação na armadura no modelo P-MIC.

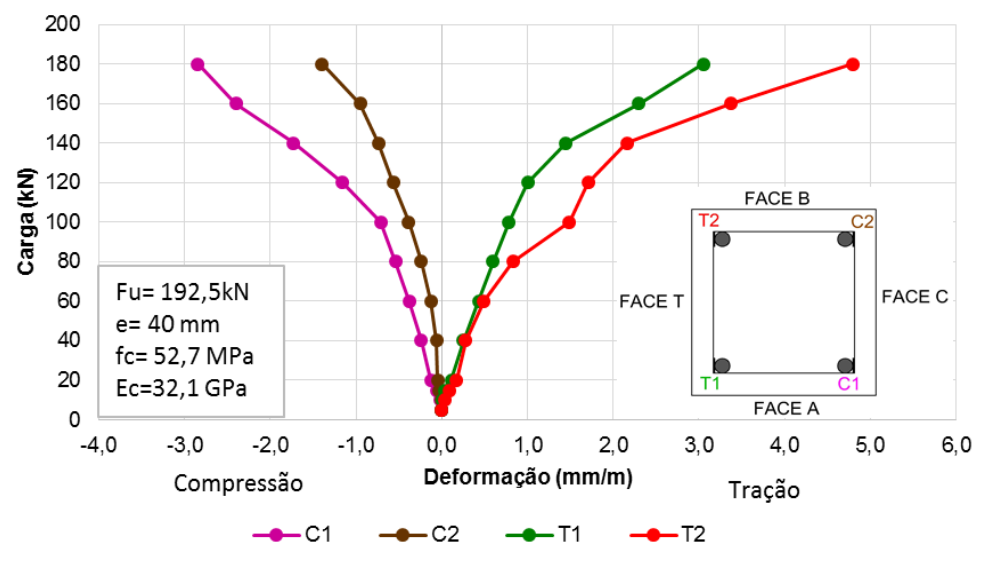

Figura 12: Diagrama Carga x Deformação na armadura no modelo P-REF.

Adotando-se comportamento tensão-deformação simétrico para o aço para a tração e compressão e lançando mão da deformação de escoamento de $2,86 \mathrm{~mm} / \mathrm{m}$, obtida experimentalmente (Ver na Tabela 3), verifica-se que, para o P-REF as armaduras T1 e T2 escoaram $(3,05 \mathrm{~mm} / \mathrm{m}$ e $4,79 \mathrm{~mm} / \mathrm{m})$ e a armadura C1 ficou em iminência de escoar $(2,85 \mathrm{~mm} / \mathrm{m})$. Quanto ao Pilar P-MIC, apenas as armaduras tracionadas escoa$\operatorname{ram}(3,54 \mathrm{~mm} / \mathrm{m}$ e $3,28 \mathrm{~mm} / \mathrm{m}$, para $\mathrm{T} 1$ e $\mathrm{T} 2$, respectivamente).

\subsection{Abertura de fissuras}

Observou-se durante o ensaio que o pilar P-REF começou a desenvolver as fissuras a partir da carga de 140 $\mathrm{kN}$; quanto ao P-MIC esta carga foi de $260 \mathrm{kN}$, revelando um comportamento de ruptura mais brusco do que o P-REF.

Finalmente, o comportamento mais rígido do pilar P-MIC em relação ao pilar P-REF, pode ser observado na grande maioria das curvas carga-deslocamento e carga-deformação mostradas ao longo dos resultados. O maior módulo de elasticidade obtido para pilar P-MIC é uma das causas prováveis. Infelizmente, pela 
ruína fora da seção intermediária ocorrida no pilar P-REF, não foi possível melhor quantificar a influência desta propriedade nos resultados obtidos.

\section{CONCLUSÕES}

As principais conclusões obtidas do presente estudo foram:

Quanto às propriedades no estado fresco, ambos os concretos se comportaram de forma semelhante, com boas condições de trabalhabilidade, medidas pelo abatimento do tronco de cone;

Quanto às propriedades mecânicas e de durabilidade dos materiais, o microconcreto apresentou melhor comportamento devido às melhores propriedades da interface pasta-agregado.

Houve um aumento de $8 \%$ no valor do Módulo elasticidade do microconcreto em relação ao concreto de referência e uma redução de 7,2\% no índice de vazios.

A expressão sugerida pela NBR 6118 [3] para a estimativa do Módulo de elasticidade estático forneceu valores ligeiramente superiores para ambos os concretos ensaiados;

Quanto aos ensaios dos pilares, a carga de ruptura do pilar executado com o microconcreto foi cerca de $13 \%$ superior ao valor da carga de ruptura obtida pelo software Esbelt 2.1; quanto ao pilar executado com o concreto de referência, esta carga de ruptura foi cerca de $30 \%$ inferior àquela obtida pelo referido software. Todavia, deve-se ressaltar que este pilar se afastou da condição de projeto, provavelmente pela ocorrência de uma excentricidade acidental devida ao desaprumo do pilar;

Os deslocamentos últimos obtidos na seção intermediária dos pilares foram cerca de $137 \%$ e $11 \%$ superiores, para o microconcreto e o concreto convencional, respectivamente, em relação ao valor obtido pelo software Esbelt 2.1, influência possível de hipóteses conservadoras adotadas pelo software e, finalmente:

Tanto pelos resultados obtidos para os materiais, quanto pelas curvas carga-deslocamento e cargadeformação obtidas experimentalmente, observou-se que o microconcreto obteve um desempenho superior em comparação ao concreto convencional, de referência. Tal fato demonstra a viabilidade técnica do uso deste material, nos quesitos analisados. Quanto ao fato de a ruptura do P-REF não ter ocorrido na seção intermediária, tal ocorrência não invalida a conclusão de que o P-MIC se comportou de forma mais rígida, posto que, ainda não sendo a seção de ruptura, as deformações no concreto do P-REF na seção intermediária foram maiores, para os mesmos níveis de carga aplicada.

\section{AGRADECIMENTOS}

Os autores agradecem à STC soluções para construção civil Ltda por ter cedido os agregados, à Grace Brasil Ltda pela doação do aditivo superplastificante, e à Votorantin cimentos, pela doação deste material. Nosso muito obrigado por suas contribuições.

\section{BIBLIOGRAFIA}

[1] ASSOCIAÇÃO BRASILEIRA DE NORMAS TÉCNICAS, Concreto - Ensaios de compressão de corpos-de-prova cilíndricos, NBR 5739, Rio de Janeiro, 2007.

[2] ASSOCIAÇÃO BRASILEIRA DE NORMAS TÉCNICAS, Projeto de estruturas de concreto - Procedimento. NBR 6118, Rio de Janeiro, 2003.

[3] ASSOCIAÇÃO BRASILEIRA DE NORMAS TÉCNICAS, Projeto de estruturas de concreto - Procedimento. NBR 6118, Rio de Janeiro, 2014.

[4] ASSOCIAÇÃO BRASILEIRA DE NORMAS TÉCNICAS, Materiais metálicos - Ensaio de tração- Parte 1: Método de ensaio à temperatura ambiente, NBR ISO 6892-1, Rio de Janeiro, 2013.

[5] ASSOCIAÇÃO BRASILEIRA DE NORMAS TÉCNICAS, Concreto e argamassa - Determinação da resistência à tração por compressão diametral de corpos de prova cilíndricos, NBR 7222, Rio de Janeiro, 2011.

[6] ASSOCIAÇÃO BRASILEIRA DE NORMAS TÉCNICAS, Concreto - Determinação do módulo estático de elasticidade à compressão, NBR 8522, Rio de Janeiro, 2008.

[7] ASSOCIAÇÃO BRASILEIRA DE NORMAS TÉCNICAS, Argamassa e concreto endurecidos - Determinação da absorção de água, índice de vazios e massa específica, NBR 9778, Rio de Janeiro, 2009.

[8] ASSOCIAÇÃO BRASILEIRA DE NORMAS TÉCNICAS, Concreto fresco - Determinação da massa específica, do rendimento e do teor de ar pelo método gravimétrico, NBR 9833, Rio de Janeiro, 2009. 
[9] ASSOCIAÇÃO BRASILEIRA DE NORMAS TÉCNICAS, Concreto - Determinação da consistência pelo abatimento do tronco de cone, NBR NR 67, Rio de Janeiro, 1998.

[10] AMERICAN CONCRETE INSTITUTE, Building code requirements for reinforced concrete, ACI 31811, Detroit: ACI, 2011.

[11] BACARJI, E. Aplicação do método dos elementos de contorno à análise de pavimentos de edifícios, São Carlos, Tese de D.Sc., Escola de Engenharia de São Carlos, Universidade de São Paulo USP, São Carlos, SP, Brasil, 2001.

[12] BORGES, A. C. L., Análise de pilares esbeltos de concreto armado solicitados a flexo-compressão oblíqua, Dissertação de M.Sc., Escola de Engenharia de São Carlos, Universidade de São Paulo, São Carlos, SP, Brasil, 1999.

[13] CAMPOS, P. E. F., Da argamassa armada ao microconcreto de alto desempenho: perspectivas de desenvolvimento para a pré-fabricação leve, Tese de D.Sc., Faculdade de Arquitetura e Urbanismo, Universidade de São Paulo, São Paulo, SP, Brasil, 2002.

[14] CHUNSHENG, Z., Li KEFEI, L., FU, M., "Numerical and statistical analysis of elastic modulus of concrete as a three-phase heterogeneous composite", Computers and Structures, v. 139, pp. 33-42, 2014.

[15] FELEKOĞLU, B., "Effects of PSD and surface morphology of micro-aggregates on admixture requirement and mechanical performance of micro-concrete", Cement \& Concrete Composites, v.29, pp.481-489, 2007.

[16] LAGERBLAD, B., KJELLSEN, K.O. Normal and high strength concretes with conventional aggregates. Engineering and transport properties of the interfacial transition zone in cementitious composites, In: RILEM TC 159-ETC and 163-TPZ,,RILEM Publications SARL, pp. 53-70, 1999.

[17] NETO, B.B.P., OLIVEIRA, D.R.C., RAMOS, D., "Efeitos do tipo, tamanho e teor de agregado graúdo no módulo de deformação do concreto de alta resistência”, Revista Matéria, v. 16, n. 2, pp. 690 - 702, 2011.

[18] ROHDEN, A. B., Efeito da resistência e da dimensão máxima característica do agregado graúdo nas propriedades mecânicas do concreto de alto desempenho, Dissertação de M.Sc., Escola de Engenharia Civil, Universidade Federal do Rio Grande do Sul, Porto Alegre, RS, Brasil, 2011.

[19] SAHMARAN, M., LACHEMI, M., HOSSAIN, K. M.A., et al., "Influence of aggregate type and size on ductility and mechanical properties of engineered cementitious composites", ACI Materials J., v. 106, n.3, pp.308-316, 2009.

[20] SCRIVENE, K., CRUMBIE, A.K., LAUGESEN, P., "The interfacial transition zone (ITZ) between cement paste and aggregate in concrete", Interface Sci,v.12, pp. 411-21, 2004.

[21] XIE, Y., CORR, D.J, JIN, F., ZHOU, H., et al., "Experimental study of the interfacial transition zone (ITZ) of model rock-filled concrete (RFC)", Cement \& Concrete Composites, v. 55, pp. 223-231, 2015. 